\title{
THE PREVALENCE OF MUSCULOSKELETAL DISORDERS AMONG DENTISTS IN THE CZECH REPUBLIC
}

\author{
Zdeňka Šustová1, Lenka Hodačová2, Martin Kapitán ${ }^{1}$
}

Charles University in Prague, Faculty of Medicine in Hradec Králové, and University Hospital Hradec Králové, Czech Republic: Department of Dentistry ${ }^{1}$; Charles University in Prague, Faculty of Medicine in Hradec Králové, Czech Republic: Department of Social Medicine ${ }^{2}$

\begin{abstract}
Summary: The aim of this study was to determine the prevalence of musculoskeletal problems among Czech dentists and to analyze the factors that affect these disorders. Information was gathered through questionnaire completed by 581 physicians. The questionnaire ascertained general information about physicians including their work habits and the characteristics of their work environment along with the occurrence of musculoskeletal problems as well as their intensity. In the past year the occurrence of at least mild difficulties associated with the locomotive system was reported by $96.9 \%$ of dentists surveyed $(n=557)$, with $66.3 \%$ of respondents $(n=381)$ reporting difficulties of a moderate or major nature. Most respondents of both sexes indicated neck pain. A statistically significant correlation with the occurrence of musculoskeletal complaints of medium and major intensity was demonstrated for the following factors: sex, age, running a private practice, past injury or musculoskeletal diseases, and the perception of work as psychologically demanding. Musculoskeletal disorders in dentists in Czech Republic are relatively frequent and serious health problem. The causes of these diseases must be identified and appropriate preventive interventions undertaken that will contribute to a reduction in the incidence of these problems.
\end{abstract}

Key words: Dentists; Musculoskeletal disorders; Questionnaire; Czech Republic

\section{Introduction}

Musculoskeletal disorders (MSDs), sometimes called disorders of the motoric system, are currently a serious problem not only in the Czech Republic, but throughout Europe. There has been an increase of MSDs in recent years and they have become a common cause of taking sick leave $(1,2)$. These disorders are due to excessive, long-term unilateral stress on muscles, tendons, joints and nerves. The problem is not only medical but also social and economic. If the dentist suffer from MSDs, the outcome of his work is decreased or he even has to take a sick leave. This can negatively affect his social and economical status.

The onset of the disorders is gradual. It is manifested in intermittent pain in the neck, back and shoulders, tingling fingers, muscle fatigue, muscle stiffness and mild limitation of full range of motions. Left untreated, the initial stages proceeds due to an accumulation of disorders and a lack of regeneration to the chronic stages which manifest by pain of varying intensity and the subsequent reduction of musculoskeletal function, negatively impacting overall health and job performance.

MSDs are classified as disorders with multifactorial etiology (3-5). Their incidence is associated with the performance of work in awkward physical positions and in adverse working conditions. About $60 \%$ of MSDs has been associated with the long-term excessive and unilateral overloading of extremities. In $35 \%$ of cases the disease has been caused by working with vibrating tools or by a combination of these risk factors (6).

Dentists rank among the professions significantly affected by the occurrence of these disorders (7-11), because their work requires small, precise movements of the fingers and hands; they often work with vibrating tools in a small working field which allows only limited mobility. To achieve the greatest visibility possible when concentrating on their work, dentists adjust their physical positions for better access to patients under their care. This means that every day for long periods of time dentists must maintain adverse static positions which result in the awkward twisting of and unilateral loading on the musculoskeletal system. The load rate is multiplied by age-related factors; it depends on the physical fitness of the doctor and is influenced by genetic predisposition (12). Another very important aspect is the psychological demands of work and stress (7).

Ergonomics is a multidisciplinary field concerned with the complex relationship between man and the working environment. Although ergonomics has penetrated into dental offices and into the manufacturing of dental equipment and instruments in recent years, the incidence of possibly preventable MSDs among dentists remains very high. Valachi has stated that two out of three dentists suffer 
from MSDs (12). A high rate of incidence of these problems among dentists is also indicated in the results of other international studies (13-18). Data on the situation among Czech dentists has thus far been lacking.

The aim of our study was thus to determine the prevalence of MSDs in Czech dentists and to analyze selected factors that may affect their occurrence.

\section{Material and methods}

The information was obtained by a questionnaire survey performed in the period from November 2010 to September 2011. Dentists voluntarily filled in questionnaires during professional educational events. The survey was anonymous. The vast majority of the completed questionnaires were returned on site during the event, but a small number of completed questionnaires were returned to us by mail at the expense of the respondents. Questionnaires were distributed to a total of 800 dentists, with the final processing including 581 completed, returned questionnaires ( $72.6 \%$ return rate).

The relatively extensive questionnaire was divided into three parts. The introductory part ascertained general information about the dentists: gender, age, height, weight and length of time of professional career. The second part of the questionnaire was devoted to the specific characteristics of the individual's working environment and work habits: working hours, body position when working and the frequency of breaks taken during the workday. An evaluation of the demands of a number of common work procedures was also included in this section. Older respondents were asked not only to complete this part of the questionnaire regarding their current situation, but also an additional section detailing their working environment and habits for the period before 1989 if they were employed in the profession of dentistry. It was not until the political system changed in the year 1989 that dentists were allowed to obtain adequate dental equipment as well as to gain experience outside of their home country. This influenced the way Czech dentists administered dental care. The last part of the questionnaire focused on the prevalence of health problems associated with the motoric system. The dentists were asked for descriptions of their health problems, including their intensity. The intensity of problems was rated on a scale of $0-3$ in which 0 meant no difficulties, 1 mild, 2 medium and 3 major problems. Data sought in the survey included the most common health problems related to MSDs that occur in dentists headaches; pain in the cervical, thoracic and lumbar spine area; pain in the shoulders, elbow and wrist; tingling fingers; pain in the hip and knee; varicose veins; and carpal tunnel syndrome. The questionnaire was partly designed based on questionnaires used in studies performed in other countries (19); additional issues concerning specific circumstances of Czech dental population were added by the authors. This article contains the part of the results about the incidence of health problems associated with the motoric system with respect to selected factors that can influence the occurrence of these disorders. The results of the evaluation concerning working environment, work habits and the difficulty of individual procedures as well as a comparison of today's situation in this country with that of before 1989 will be contained in subsequent publications.

Data analysis was performed using the program NCSS 2007. The methods used were descriptive statistics and the chi-square test for independence in the contingency tables. The threshold for statistical significance was set at $\mathrm{p}=0.05$.

\section{Results}

Of the 581 respondents, 163 were men $(28.1 \%)$ and 418 women $(71.9 \%)$. The sample obtained was compared with the entire population of dentists in the Czech Republic in terms of gender, age and expertise. The data characterizing the current population of Czech dentists was obtained from the Czech Dental Chamber (CDC) Yearbook 2010, issued by the CDC in 2011 (20). According to the statistics listed in this publication, $35 \%$ of Czech dentists were men and $65 \%$ women. Difference when comparing this data with our set was statistically significant $(\mathrm{p}<0.001)$.

The average age of our sample of respondents was 46.5 years, the median 51 years, and the age range varied from 24 to 77 years of age. A comparison of the percentages of the sample with the total population of Czech dentists in each age category is listed in Table 1 . The differences in the age representation of physicians in our group as opposed to the total population of Czech dentists were statistically significant $(\mathrm{p}<0.001)$.

Table 1: Age categories of the general population of dentists in the Czech Republic compared with survey respondents

\begin{tabular}{|l|c|c|}
\hline \multicolumn{1}{|c|}{ Age category } & CR (\%) & Sample $\mathbf{( \% )}$ \\
\hline 24-29 years & 12.2 & 18.4 \\
\hline 30-34 years & 6.9 & 7.1 \\
\hline 35-39 years & 6.6 & 8.3 \\
\hline 40-44 years & 7.3 & 7.2 \\
\hline 45-49 years & 6.2 & 7.1 \\
\hline 50-54 years & 13.2 & 10.8 \\
\hline 55-59 years & 26.7 & 28.7 \\
\hline 60-64 years & 12.0 & 8.8 \\
\hline 65-69 years & 7.0 & 2.9 \\
\hline 70-99 years & 1.9 & 0.7 \\
\hline
\end{tabular}

In terms of the representation of individual specializations, the set consisted of $93.1 \%$ general dental practitioners $(n=539)$ and $6.9 \%$ specialists $(n=42)$, of which $6.0 \%$ were orthodontists $(n=36)$ and $0.9 \%$ maxillofacial 
surgeons $(n=6)$. There was not a statistically significant difference in our representation of specializations when compared with a total population of dentists. The average length of professional career in dentistry was 21.5 years and the median 25 years, ranging from 1 to 53 years of practice.

The average height of male respondents was $180.4 \mathrm{~cm}$, of females $167.7 \mathrm{~cm}$. The average male body weight was $85.6 \mathrm{~kg}$, females $66.2 \mathrm{~kg}$. Average values of body mass index (BMI) were 26.3 for men and for women 23.6. According to the statistics for the Czech Republic reported in the European Health Interview Survey EHIS CR 2008, the average height of Czech men was $177.7 \mathrm{~cm}$ and of women $165.3 \mathrm{~cm}$; average body weight in males was $83.6 \mathrm{~kg}$ and in females $66.2 \mathrm{~kg}$; the average BMI in men and in women were 26.5 and 25.4 respectively (21).

The assessment of the incidence of health complaints showed that in the last year $96.9 \%$ of the respondents ( $\mathrm{n}$ $=557$ ) indicated at least one kind of the problems listed regardless the intensity. During the last year only 18 respondents reported having absolutely no health problems, which was $3.1 \%$ of our sample (6 respondents didn't determine whether or not they had problems). When the sample was evaluated in terms of the intensity of the health problems indicated, the first group of physicians showed problems of only mild intensity or no difficulty at all. This set comprised $33.7 \%$ of the respondents $(n=194)$ surveyed. The second group consisted of the respondents who rated their difficulties as a medium to major (the group of dentists with problems). We believe that, in addition to lifestyle factors, the dentists in the second group may be also negatively affected by factors associated with the profession of dentistry, so for this reason we have focused to a greater extent on this group.

From the total number of respondents, $66.3 \%(n=381)$ of the physicians reported problems. Of the men, $58.4 \%$ $(\mathrm{n}=94)$ indicated medium or major problems, and $69.3 \%$ $(\mathrm{n}=287)$ of the women. This difference was statistically significant $(p=0.0127)$. A statistically significant influence was demonstrated for age $(p<0.001)$, while on the other hand the specialization of the physicians had no affect on the incidence of medium and major problems $(p=0.333)$. Owner-operators of private practices reported medium and major problems significantly more frequently $(p<0.001)$ than the dentists employed in these private establishments.

The incidence of MSDs in the blood relatives of those surveyed or the occurrence of musculoskeletal congenital defects in our group had no statistically significant effect on the incidence of medium and major problems $(p=0.0748$ and 0.553 ). In respondents with a history of serious disease in or injury to the musculoskeletal system, medium and major problems were reported significantly more often ( $p=0.005$ and 0.028 ). Participating regularly at least once a week in sport activities or even less frequently had no statistically significant effect $(p=0.601$ and 0.192). Smoking during working hours also did not significantly affect the incidence of medium and major problems $(p=0.177)$.
Of physicians who subjectively complained of medium and major problems, exercise was reported to be used as a therapy in $86.9 \%(n=331)$ of the responses; $48.6 \%$ $(\mathrm{n}=185)$ indicated prescription of medications for themselves; $46.7 \%(n=178)$ sought out the help of a specialist. Workload had to be reduced during the last year for $22.6 \%$ $(n=86)$ of the doctors with medium and major difficulties. Sick leave for these difficulties was taken by $4.5 \%(n=17)$ of the dentists during the last year. The number of practitioners reporting medium or major problems who underwent operative intervention was $5.8 \%(n=22)$.

When asked whether they consider their work in the last year to be psychologically demanding, $77.8 \%$ of the surveyed physicians $(n=444)$ responded positively. Dentists who reported difficulties of medium and major intensity perceived their work as psychologically demanding significantly more often $(\mathrm{p}<0.001)$ as compared with physicians who indicated no or only mild musculoskeletal problems (Table 2). Physicians who in the last year considered their work psychologically demanding reported a significantly greater frequency of headaches $(\mathrm{p}=0.0434)$.

Table 2: Relationship between subjective assessment of psychological demands of work and the incidence of medium and major problems

\begin{tabular}{|l|c|c|}
\hline & \multicolumn{2}{|l|}{$\begin{array}{l}\text { Do you consider your work to } \\
\text { be psychologically demanding } \\
\text { in the last year? }\end{array}$} \\
\hline $\begin{array}{l}\text { Medium or major } \\
\text { problems }\end{array}$ & $\begin{array}{c}\text { Yes } \\
\mathrm{n}(\%)\end{array}$ & $\begin{array}{c}\text { No } \\
\mathrm{n}(\%)\end{array}$ \\
\hline Yes & $314(82.6)$ & $66(17.4)$ \\
\hline No & $130(68.1)$ & $61(31.9)$ \\
\hline Total & $444(77.8)$ & $127(22.2)$ \\
\hline
\end{tabular}

A total of $95.8 \%$ of all physicians surveyed $(n=549)$ worked mainly with their right hand, with only $2.6 \%$ $(n=15)$ being predominantly left-handed. These factors had no statistically significant effect on the incidence of medium and major problems ( $\mathrm{p}=0.985$ and 0.589$)$.

Eighty-eight point eight percent $(n=494)$ of the physicians surveyed had previously received information about ergonomics. This, however, had no statistically significant effect on the rate of medium and major problems with $\operatorname{MSDs}(\mathrm{p}=0.562)$.

The incidence of different types of health problems and their intensity is given in Table 3 . Figure 1 compares the incidence of various types of health problems in men and women without consideration of their intensity. The results shown in Figure 2 suggest how the respondents evaluated their own current health status. Of the dentists surveyed, $68 \%(n=389)$ considered their health to be "good" or "very good". 
Table 3: Incidence of various health problems and their severity

\begin{tabular}{|l|c|c|c|c|}
\hline & $\begin{array}{c}\text { No } \\
\mathbf{n}(\mathbf{\%})\end{array}$ & $\begin{array}{c}\text { Mild } \\
\mathbf{n}(\mathbf{\%})\end{array}$ & $\begin{array}{c}\text { Medium } \\
\mathbf{n}(\mathbf{\%})\end{array}$ & $\begin{array}{c}\text { Major } \\
\mathbf{n}(\mathbf{\%})\end{array}$ \\
\hline Headache & $227(39.5)$ & $229(39.8)$ & $101(17.6)$ & $18(3.1)$ \\
\hline Neck pain & $126(21.9)$ & $221(38.4)$ & $185(32.2)$ & $43(7.5)$ \\
\hline Upper back pain & $289(50.3)$ & $130(22.6)$ & $135(23.5)$ & $21(3.7)$ \\
\hline Lower back pain & $144(25.0)$ & $195(33.9)$ & $173(30.1)$ & $63(11.0)$ \\
\hline Shoulder pain & $275(47.8)$ & $149(25.9)$ & $111(19.3)$ & $40(7.0)$ \\
\hline Elbow pain & $412(71.7)$ & $107(18.6)$ & $46(8.0)$ & $10(1.7)$ \\
\hline Wrist pain & $352(61.2)$ & $138(24.0)$ & $65(11.3)$ & $20(3.5)$ \\
\hline Pain / tingling fingers & $342(59.5)$ & $130(22.6)$ & $77(13.4)$ & $26(4.5)$ \\
\hline Hip pain & $344(59.8)$ & $149(25.9)$ & $65(11.3)$ & $17(3.0)$ \\
\hline Knee pain & $358(62.3)$ & $127(22.1)$ & $67(11.7)$ & $23(4.0)$ \\
\hline Varicose veins & $371(64.5)$ & $113(19.7)$ & $67(11.7)$ & $24(4.2)$ \\
\hline Carpal tunnel syndrome & $491(85.4)$ & $50(8.7)$ & $26(4.5)$ & $8(1.4)$ \\
\hline
\end{tabular}

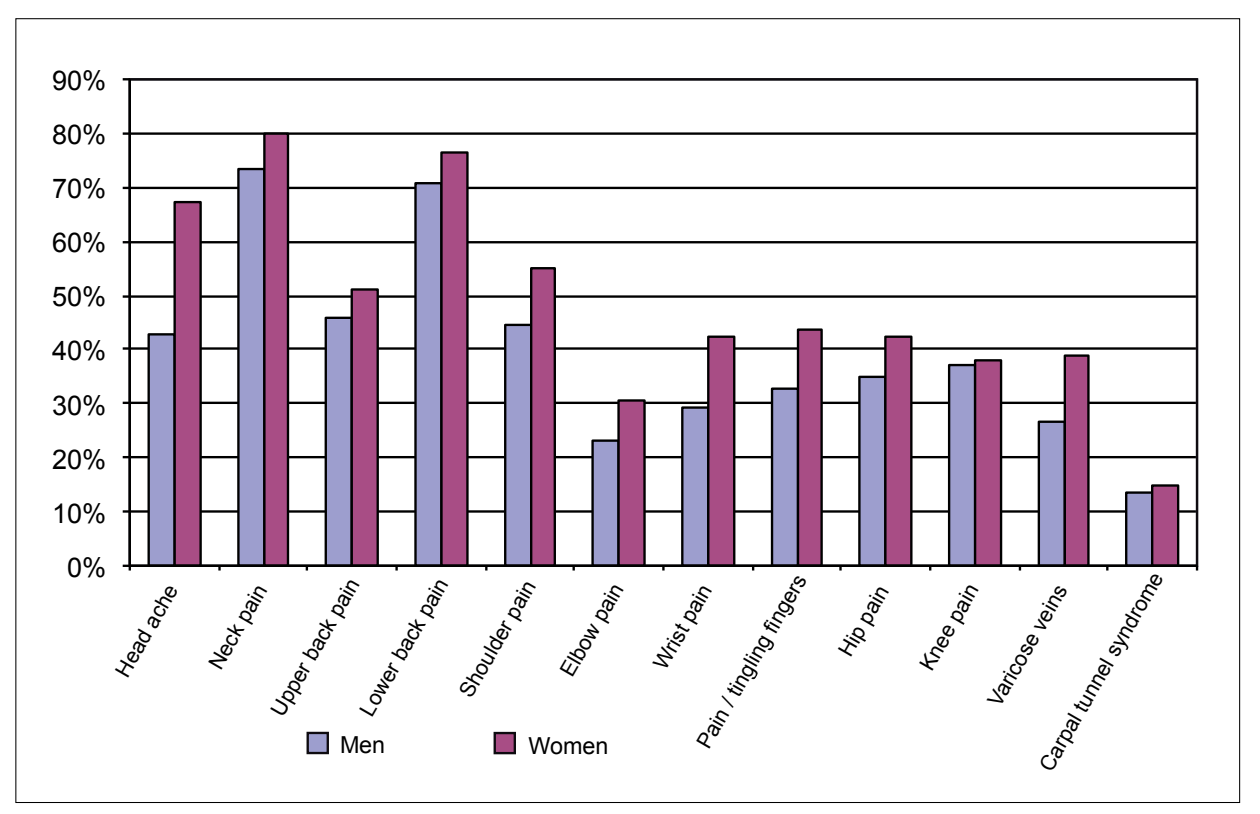

Fig. 1: Comparison of the incidence of health problems in men and women without consideration of their intensity

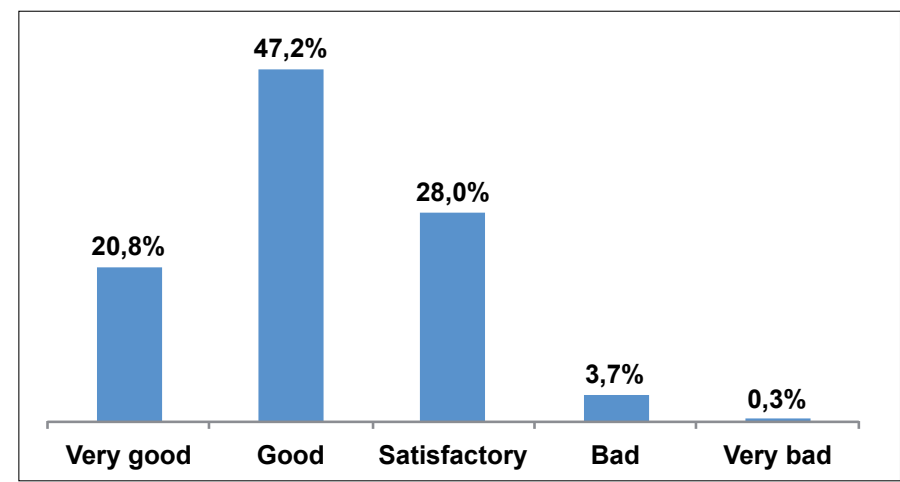

Fig. 2: Self-evaluation of the current health status of the respondents 


\section{Discussion}

The data of the sample of dentists in our survey has been compared with a large number of similar studies from other countries, where the problem has been dealt with to a greater extent $(1,11,14,16,17)$. Compared with the whole population of Czech dentists there were statistically significantly less men in our sample. This is likely due to less participation by male dentists in training events as well as their lesser willingness to fill out questionnaires.

The average age of physicians in our group was compared with the total population of dentists in the Czech Republic. The age of our group proved to be significantly lower, representing a greater frequency of respondents aged 24 to 29 years and lower rates in the categories of respondents at 65 to 69 and 70 to 99 years of age. We obtained a significant percentage of the completed questionnaires at educational events in various fields of dentistry. These are offered especially to younger dentists in the context of lifelong education. Dentists over the age of 60 usually attend these events less frequently and generally more often seek out colleagues at meetings of specialists. Although in terms of age and sex the sample of respondents can not be considered representative of the entire population of dentists in the Czech Republic, we consider the results of the survey important, as this is the first study of its kind in the country. Despite the fact that in our set younger physicians were over-represented, we must conclude that the percentage of difficulties shown among dentists (especially women) were relatively high. Results of a number of foreign studies show that difficulties associated with the motoric system - primarily in the neck, thoracic spine and shoulder regions - have been indicated to a great extent, not only by young doctors in their first years of work but also by dental students during their studies $(3,8,14,17)$. In the absence of the timely correction and elimination of the negative factors, they are compounded by advancing age and continuing years of working practice. This in turn leads to a marked decrease in the ability to adapt to these health problems, thus also increasing their intensity. Combined with the existence of other associated medical problems, the likely result is a deterioration of the physician's overall state of health as well as a negative impact on job performance and on the doctor's own sense of wellbeing (8).

Further, the average BMI for men indicated that most were overweight. This factor may influence the onset of MSDs and increase their intensity as well as contribute to the emergence of non-infectious chronic disorders or types of lifestyle diseases (22).

Compared with the national statistics, the height of men and women in our group, ranging from 150 to $205 \mathrm{~cm}$, was above average.

Knowledge of the principles of ergonomics can assist dentists in establishing or upgrading their own practice. The right kind of equipment should be set up, enabling doctors to work in line with ergonomic principles. This is particularly important for taller physicians, especially in the case of great height differences between the doctor and assistant, as well as for left-handed clinicians.

In our questionnaire survey the vast majority of dentists $(96.9 \%)$ reported the existence of at least one of the listed health problems in mild, medium or major intensity during the last year. It is widely known that musculoskeletal problems of mild intensity are very common in the current adult population at large. These relate mainly to sedentary lifestyle, lack of physical activity, improper diet and an increased percentage of those who are overweight or obese. In the analysis of our results, therefore, we focused more on respondents who reported difficulties of medium or major intensity. We expected not only the negative influence of lifestyle, but also factors associated with the profession of dentistry. Difficulties of at least medium intensity were reported in our sample by a total of $66.3 \%$ of the physicians, a number which corresponds to results from similar studies abroad. Thus it can be stated that the Czech Republic is roughly average in terms of the rate of occurrence of MSDs among dentists $(1,23)$. Women dentists in our study reported statistically significantly more difficulties of medium and major intensity, a fact which has also been frequently observed in foreign studies $(8,17,24)$.

Health problems with the neck were most prevalent in both sexes in our sample, closely followed by problems with the lumbar spine region. The nature of these problems corresponds to the overloading of the spine at work, where dentists sit with their head bent excessively and their body in awkward positions as well as stand with their lumbar spine twisted in unnatural postures. Shoulder pain in women occurred at a rate of $55.1 \%$ and in men $44.7 \%$. While working, the dentist remains in awkward positions for a long time and repeatedly. If the recommended principles of ergonomics are ignored in terms of patient positioning and the use of appropriate instruments, the shoulders are often overloaded during the extended periods of arm elevation and leaning forward in sitting or standing positions. The higher incidence of shoulder pain in women can be attributed to the more limited physical strength in their shoulder muscles. Headaches in women were listed as the third most frequent complaint, in men the fifth most common. The increased incidence of headaches in women was apparently associated with cervical spine pain in combination with psychosocial factors and stress. This has been associated with a lower threshold for pain and the lower endurance of women to physical exertion and stress (8).

The significantly higher incidence of problems in operators of dental practices and the lower rates for their employees can be attributed to the fact that such employees are mostly younger doctors, while the operators of dental practices in the Czech Republic fall within the higher age categories, often being of retirement age. It may also reasonably be assumed that the operators of dental practices often work for longer periods in excess of standard working hours. 
The finding that previous illness or musculoskeletal injury significantly increased the incidence of medium and major problems can serve as a call to dentists to reflect on this fact when balancing the workload in the office with sports and other activities outside of it. In our survey occasional or regular participation in sport had no statistically significant effect on the incidence of medium and major problems. On the other hand, if implemented in good time and correctly, exercises targeted to strengthen flaccid muscles - especially in the back and abdomen - in combination with relaxation exercises to soothe overloaded muscles are important components in the prevention of MSDs.

In the questions about smoking during working hours, we wanted to verify the assumption that smoking breaks lead to an interruption of work activities and thus would allow doctors to rest for a short while in a different body position. The effect on the incidence of MSDs, however, was insignificant. More than $87 \%$ of the physicians reported that they dealt with their problems by exercising; less than half of physicians surveyed sought professional help. Difficulties of medium or major intensity caused a decrease in the workload for every fifth dentist in our group, but in the last year only $5 \%$ of physicians reported drawing paid sick leave. This reflects not only a sense of responsibility to patients and other members of the team, but also a concern about the threat to prosperity and loss of clientele leads dentists to maintain the practice even if they must decrease their working load. Also the fact that nearly six dentists out of every hundred respondents underwent an operation for medium or major difficulties of the motoric system should not be overlooked.

Approximately three quarters of respondents considered their work psychologically demanding. Physicians who reported difficulties of medium and major intensity considered their work psychologically demanding at a rate of $82 \%$. It is known that stress increases muscle tension in a way similar to physical exercise and in combination with adverse psychosocial factors stress can affect the incidence and intensity of musculoskeletal problems, especially in the area of the neck, shoulders and upper limbs $(7,8,13,25)$.

Interesting results also emerged from the answers to the question of how doctors themselves assessed their current health status, with $68 \%$ of respondents considering their health to be "good" or "very good." A comparable percentage of physicians $(66.3 \%)$, however, reported at least one of the listed ailments at medium or major intensity. Subjective perceptions and feeling oneself healthy is an important factor that affects the quality of life of individuals (26), but this feeling should not lead to an underestimation of the primary symptoms of MSDs. Long-term pain, muscle stiffness, loss of grip strength and restrictions of movement can not simply be taken for granted as inevitable in the profession of dentistry. In addition the feeling of being generally healthy even in the presence of certain health problems contributes to individual perceptions about pain intensity. The profession of dentistry has been chosen as an independent occupation, it is relatively well-paid and accompanied by high social status and moreover doctors can expect good prospects after their active work years have been completed. These positive factors certainly can at least partially compensate for existing health problems and lead to a subjective perception of overall good health.

\section{Conclusions}

The questionnaire survey showed that MSDs are a relatively common health problem among dentists in the Czech Republic with a high prevalence. Most respondents of both sexes complained most often of neck pain, followed by pain in the lumbar spine region. It is necessary to continue to identify the causes of the disorders and subsequently focus on appropriate preventive interventions that can help to reduce the prevalence of these problems, because this problem is not only medical but also social and economic.

\section{Acknowledgements}

The authors would like to thank all the dentists who completed the questionnaires as well as Dr. Eva Čermáková for her help with the statistical processing of our data and Mr. Daniel Paul Sampey, MFA for the language revision of the article.

Supported by the programme PRVOUK P37/13/550, PRVOUK P37/09.

\section{References}

1. Alexopoulos EC, Stathi IC, Charizani F. Prevalence of musculoskeletal disorders in dentists. BMC Musculoskeletal Disorders 2004; 5(9): 16. DOI: 10.1186/1471 -2474-5-16. Available from http://www.biomedcental.com/1471-2474/5/16.

2. European Agency for Safety and Health at Work. Work-related musculoskeletal disorders in the EU-Facts and figures. Luxembourg: Publications Office of the European Union 2010; 179 p. DOI: 10.2802/10952.

3. Puriene A, Janulyte V, Musteikyte M, Bendinskaite R. General Health of dentists. Literature review. Stomatologija 2007; 9(1): 10-20.

4. Valachi B, Valachi K. Mechanisms leading to musculoskeletal disorders in dentistry. J Am Dent Assoc 2003; 134(10): 1344-50.

5. Vacek J, Pohanka M, Siegelova J. Statistické hodnocení efektivity léčby bolestivých stavů lumbosakrální oblasti. Rehabil Fyz Lék 2011; 18(3): 111-19. [in Czech]

6. Marek J, Skrehot P. Základy aplikované ergonomie. Praha: VÚBP v.v.i, 2009; 118. [in Czech]

7. Lindfors P, von Thiele U, Lundberg U. Work Charakteristics and Upper Extremity Disorders in Female dental health Workers. J Occup Health 2006; 48(3): 192-7.

8. Puriene A, Aleksejuniene J, Petrauskiene J, Balciuniena I, Janulyte V. Self-reported Occupational health Issues among Lithuanian Dentists. Ind Health 2008; 46(4): 369-74. DOI: 10.2486/indhealth.46.369.

9. Leggat PA, Kedjarune U, Smith DR. Occupational Health Problems in Modern dentistry: A Review. Ind Health 2007; 45(5): 611-21. DOI: 10.2486 /indhealth.45.611.

10. Sadig W. Ergonomics in dental practice. Pakistan Oral \& Dent J 2000; 20(2): 205-13.

11. Chowanadisai S, Kukiattrakoon B, Yapong B, Kedjarune U, Leggat PA. Occupations health problems of dentists in Southern Thailand. Int Dent J 2000; 50(1): 36-40.

12. Valachi B. The Journey Begins. In: Valachi B. Practice Dentistry Pain-Free: Evidence-Based Strategies to Prevent Pain and Extend Your Career. 1st ed. Portland: Posturedontics Press; 2008. p. 1-20.

13. Myers HL, Myers LB. "It's difficult being a dentist": stress and health in the general dental practicioner. Br Dent J 2004; 197(2): 89-93.

14. Leggat PA, Smith DR. Musculoskeletal disorders self-reported by dentists in Queensand, Australia. Aust Dent J 2006; 51(4): 324-7. 
15. Wunderlich M, Eger T, Ruther T, Meyer-Felcke A, Leyk D. Analysis of spine loads in dentistry-impact of an altered sitting position of dentists. J Biomedical Science and Engineering 2010; 3: 664-71. DOI: 10.4236/jbise.2010.37090.

16. Rundcrantz BL, Johnsson B, Moritz U. Cervical pain and discomfort among dentists. Epidemiological, clinical and therapeutic aspects. Swed Dent J 1990; 14(2): 71-80.

17. Sharma P, Golchha V. Awareness among Indian dentists regarding their role of physical activity in prevention of work related musculoskeletal disorders. Indian J Dent Res 2011; 22(3): 381-4.

18. Szymanska J. Disorders of the musculoskeletal system among dentists from the aspect of ergonomics and prophylaxis. Ann Agric Environ Med 2002; 9(2): 169-73.

19. Kuorinka I, Jonsson B, Kilbom A,Vinterberg H, Biering-Sorensen F, Andersson G, et al. Standardised Nordic questionnaires for the analysis of musculoskeletal symptoms. Appl Ergon 1987; 18(3): 233-7. DOI: 10.1016/0003-6870(87)90010-X.

20. Česká stomatologická komora. Ročenka ČSK 2010. (online) ČSK Praha 2011;3. (cited 2012 May 11) Available from www.dent.cz/en/img_data/.../Rocenka 2010 na\%20web_komplet-DEF.pdf. [in Czech]

21. ÜZIS ČR. European Health Interview Survay in CR - EHIS CR Body Mass
Index, physical activity, consumption of fruits and vegetbles. (online) Úzis CR: Praha, Aktuální informace 2010; 37. (cited 2012 May 8) Available from www.uzis.cz/.../european-health-interview-survey-czech-republic-ehis-2008. [in Czech]

22. Sethi J, Sandhu JS, Imbanathan V. Effect of Body Mass Index on work related musculoskeletal discomfort and occupational stress of computer workers in a developed ergonomic setup. Sports Med Arthrosc Rehabil, Ther Technol 2011; 3(1): 22. DOI 10.1186/1758-2555-3-22.

23. Hayes M, Cockrell D, Smith DR. A systematic review of musculoskeletal disorders among dental professionals. Int J Dent Hyg 2009; 7(3): 159-65. DOI: 10.1111/j.1601-5037.2009.00395.x.

24. Valachi B. Musculoskeletal health of the woman dentists: Distincive interventions for a growing population. J Calif Dent Assoc 2008; 36(2): 127-32.

25. Bongers PM, Kremer AM, Laak J. Are psychosocial factors, risk factors for symptoms and signs of the shoulder, elbow or hand/wrist? A review of the epidemiological literature. Am J Ind Med 2002; 41(5): 315-42. DOI: 10.1002 ajim. 10050

26. Hodačová L, Šmejkalová J, Čermáková E, Slezák R, Jacob V, Hlaváčková E. Oral Health-related Quality of Life in Czech population. Cent Eur J Public Health 2010; 18(2): 76-80.

Received: 20/07/2013

Accepted in revised form: 21/11/2013

\section{Corresponding author:}

MUDr. Zdeňka Šustová, Department of Dentistry, University Hospital, Sokolská 581, 50005 Hradec Králové, Czech Republic; email: zdenka.sustova@fnhk.cz 